02,12

\title{
Влияние сильного межузельного кулоновского взаимодействия на топологические свойства сверхпроводящей нанопроволоки
}

\author{
(C) М.С. Шустин, С.В. Аксенов
}

Институт фризики им. Л.В. Киренского, ФИЦ КНЦ СО РАН,

Красноярск, Россия

E-mail: mshustin@yandex.ru

Поступила в Редакцию 8 июля 2021 г.

В окончательной редакции 13 июля 2021 г.

Принята к публикации 16 июля 2021 г.

\begin{abstract}
Для сверхпроводящей нанопроволоки со спариванием расширенного $s$-типа симметрии спин-орбитальным взаимодействием Рашбы в магнитном поле проанализировано влияние сильных межузельных зарядовых корреляций на одночастичные майорановские возбуждения. Данная проблема исследована на основе численного метода ренорм-группы для матрицы плотности. Показано, что с ростом интенсивности отталкивания электронов, находящихся на соседних узлах, в нижней хаббардовской зоне открытой системы реализуются две подзоны. На основе расчетов майорановской поляризации и вырождения спектра запутанности обнаружено, что топологически нетривиальная фаза с одним краевым состоянием реализуется на том краю каждой из подзон, где концентрация электронов или дырок минимальна.
\end{abstract}

Ключевые слова: сверхпроводящие нанопроволоки, майорановские моды, сильные электронные корреляции.

DOI: $10.21883 /$ FTT.2021.11.51573.23s

\section{1. Введение}

Современные эксперименты с гибридными сверхпроводящими нанопроволоками $(\mathrm{CП})$, которые характеризуются наличием сильной спин-орбитальной связи и большими значениями $g$-фактора, не дают однозначного положительного ответа на вопрос о реализации на концах этой структуры майорановских мод (ММ) $[1,2]$. В результате возникает потребность в более детальном изучении явления топологической сверхпроводимости [3-5] и свойств майорановских состояний, например, его нелокальности [6-8], спиновой поляризации [9-12], особенностей магнитокалорического эффекта $[13,14]$.

Одна из важных проблем - вопрос о влиянии кулоновских взаимодействий на майорановские связанные состояния (МСС). В большей части теоретических исследований этих квазичастичных возбуждений рассматриваются квадратичные гамильтонианы, для которых была дана классификация фаз топологической сверхпроводимости $[15,16]$. Однако при непосредственном рассмотрении СП полная экранировка кулоновских взаимодействий в полупроводниковой нанопроволоке массивном сверхпроводником в общем случае не является гарантированной [17]. В результате более качественное описание топологических свойств гибридной наноструктуры должно включать как наведенное за счет эффекта близости куперовское спаривание, так и процессы многочастичного рассеяния.

Стоит отметить, что действие электрических полей электродов затвора, количество которых в современных экспериментах по детектированию МСС может быть весьма значительным, также способно существенно влиять на интенсивность зарядовых корреляций в полупроводниковой проволоке. В частности, в работе [18] было показано, что зависимость тока через проволоки InAs от напряжения смещения и температуры демонстрирует скейлинговое поведение в соответствии с теорией жидкости Томонаги-Латтинжера (несмотря на перемешивание спиновых и зарядовых степеней свободы за счет сильной спин-орбитальной связи) [19-21]. Полученный параметр электрон-электронного взаимодействия указывает на реализацию режима сильных электронных корреляций (СЭК) при низких концентрациях носителей в проволоке.

Одним из факторов, препятствующих обнаружению MCC в проволоках, является подавление магнитным полем сверхпроводимости подложки или внешней оболочки гибридной наноструктуры. Этой проблемы можно избежать, если предположить, например, что куперовское спаривание в СП индуцировано за счет эффекта близости с нетрадиционным сверхпроводником. Тогда в нулевом магнитном поле и сохранении симметрии относительно обращения времени возникают крамерсовские пары МСС (т. е. пары ММ на обоих концах проволоки) [22]. В случае с наведенным $s$-волновым спариванием такие возбуждения можно получить, рассмотрев две проволоки со спин-орбитальным взаимодействием, находящиеся на противоположных поверхностях традиционного сверхпроводника. В результате топологически нетривиальная фаза имеет место, если главный вклад в создание куперовской неустойчивости дают процессы перекрестного андреевского отражения, а не парное туннелирование электронов в одну из проволок. Это 
условие выполняется именно в случае сильного хаббардовского отталкивания [23]. Отмечалось также, что в квазиодномерных СП относительно большая величина кулоновского отталкивания способна вызывать смену знака эффективного потенциала куперовского спаривания и также приводить к возникновению крамерсовских пар МСС [24]. Также СЭК могут приводить к реализации парафермионов в системах с СП $[25,26]$.

Существенно, что учет сильных электронных взаимодействий встречает фундаментальные теоретические трудности, связанные со значительной перенормировкой эффективных взаимодействий, изменением топологической классификации [27], а также корректным определением и построением операторов ММ. Так, многочастичные операторы ММ были построены, в том числе аналитически, и подробно исследованы в случае модели цепочки Китаева [28-30]. Показано, что одним из следствий существования „многочастичного“ МСС является устойчивость $4 \pi$-эффекта Джозефсона по отношению к межузельным кулоновским взаимодействиям в случае, если гамильтониан всей системы (две туннельно связанные цепочки Китаева) обладает электрондырочной симметрией [31]. Также, в работе [32] для спиновой 1D XYZ-модели, которая может быть сведена к цепочке Китаева со взаимодействиями, также был построен оператор возбуждения майорановского типа в выделенной точке параметров системы.

Значимость проблемы многочастичных взаимодействий также связана с реализацией топологических квантовых вычислений. Несмотря на фундаментальную устойчивость МСС по отношению к локальным возмущениям [33], различные процессы сбоя фазы все же способны приводить к разрушению состояния топологического кубита (например, из-за гибридизации волновых функций ММ в коротких проволоках) [34]. В качестве одного из источников декогеренции могут выступать флуктуации электростатического потенциала электродов затвора [35]. В свою очередь, в ряде работ и в рамках среднеполевого описания и на основе метода ренормгруппы для матрицы плотности (DMRG) было обнаружено, что как одноузельные [36], так и межузельные [37] зарядовые корреляции при определенной интенсивности могут приводить к увеличению устойчивости МСС по отношению к различным процессам декогеренции. Однако, в противоположность этому, точная диагонализация гамильтонианов коротких цепочек Китаева показывает, что учет взаимодействий в дальних координационных сферах может уменьшать время жизни МСС [38].

Влияние зарядовых корреляций на топологические фазы в рамках метода DMRG наиболее часто рассматривается на примере модели Китаева [39], а также сводящейся к ней в сильных магнитных полях модели СП со спин-орбитальной связью [40]. Гамильтониан последней относится к симметрийному классу D, когда возможны только две фазы: тривиальная с числом Майорана (топологическим инвариантом) $M=+1$ и нетривиальная с $M=-1$. В работах $[41,42]$ анализировалось влияние электрон-электронных взаимодействий в СП симметрийного класса BDI. Последнее означает возможность реализации нескольких топологически нетривиальных фаз: фазы с одной парой MМ на концах структуры (аналогично СП класса D) и фаза с двумя МCC.

В упомянутых исследованиях $[41,42]$ основное внимание уделялось фактору хаббардовского отталкивания. B настоящей работе с помощью алгоритма DMRG pacсматривается вопрос о влиянии сильного межузельного отталкивания электронов на топологические свойства сверхпроводящей проволоки симметрийного класса BDI.

\section{2. Модель и метод}

Рассмотрим модель СП, учитывающую ряд взаимодействий: спин-орбитальную связь Рашбы, кулоновское отталкивание электронов и зеемановское расщепление. В приближении сильной связи гамильтониан такой системы, содержащей $N$ узлов, имеет вид $[40,41]$ :

$$
\begin{aligned}
H= & \sum_{f=1 ; \sigma}\left[\xi_{\sigma} a_{f \sigma}^{+} a_{f \sigma}+\Delta a_{f \uparrow} a_{f \downarrow}+\Delta^{*} a_{f \downarrow}^{+} a_{f \uparrow}^{+}+\frac{U}{2} n_{f \sigma} n_{f \bar{\sigma}}\right] \\
& -\sum_{f=1 ; \sigma}^{N-1}\left(\frac{t}{2} a_{f \sigma}^{+} a_{f \sigma}+\frac{\alpha}{2} \eta_{\sigma} a_{f \sigma}^{+} a_{f+1 \bar{\sigma}}\right. \\
& \left.-\Delta_{1} a_{f \sigma} a_{f+1, \bar{\sigma}}-\frac{V}{2} \sum_{\sigma^{\prime}} n_{f \sigma} n_{f+1 \sigma^{\prime}}+\text { h.c. }\right)
\end{aligned}
$$

Содержащиеся здесь слагаемые описывают одномерную систему фермионов с интегралом перескока $t / 2$, параметром спин-орбитального взаимодействия Рашбы $\alpha / 2$, а также зависящую от проекции спина энергию фермиона на одном узле (отсчитанную от химпотенциала $\mu): \xi_{\sigma}=-\mu+\eta_{\sigma} h, h=g \mu_{\mathrm{B}} H / 2, g-$ фактор Ланде, $\mu_{\mathrm{B}}-$ магнетон Бора, $H-$ эффективное магнитное поле, сформированное внешним магнитным полем или наведенными магнитными корреляциями. $a_{f \sigma}\left(a_{f \sigma}^{+}\right)-$ оператор уничтожения (рождения) фермиона на узле с номером $f$ и проекцией спина $\sigma=\uparrow, \downarrow ; \eta_{\uparrow}=1$, $\eta_{\downarrow}=-1$. Слагаемые с амплитудами $\Delta$ и $\Delta_{1}$ связаны с существованием в нанопроволоке (за счет эффекта близости) потенциала сверхпроводящего спаривания расширенного s-типа симметрии. Также приведены слагаемые гамильтониана, соответствующие учету одноузельного $(U)$ и межузельного $(V)$ кулоновского взаимодействия фермионов. Оператор числа электронов на узле $n_{f \sigma}=a_{f \sigma}^{+} a_{f \sigma}$. Энергетические параметры модели (1) считаются действительными и измеряются в единицах $t>0$.

Исследование равновесных свойств модели (1) в режиме СЭК $(U, V \gg 1)$ осуществлялось методом DMRG [43-45]. Его основная идея аналогична используемым в других ренорм-групповых подходах [46] и состоит в последовательном исключении из рассмотрения набора состояний исходного микроскопического гамильто- 
ниана. В результате анализируется эффективная модель, которая, тем не менее, описывает главные физические свойства первоначальной.

Реализуемый в работе алгоритм DMRG включал несколько этапов:

1. На первом этапе рассматривался левый кластер $\mathrm{L}$ из $N_{0}$ узлов, все $M_{\mathrm{L}}$ собственных состояний которого могут быть получены точно. В настоящей работе в качестве гамильтониана такого кластера использовался оператор (1) с тремя узлами: $H_{\mathrm{L}}=\left.H\right|_{N \rightarrow N_{0}=3}$. Собственные состояния гамильтониана $H_{\mathrm{L}}$ образуют гильбертово пространство, $\left\{\left|m_{\mathrm{L}}\right\rangle\right\}$, состоящее из $M_{\mathrm{L}}=64$ состояний. Аналогичным образом формируется пространство состояний $\left\{\left|m_{\mathrm{R}}\right\rangle\right\}$ правого кластера $\mathrm{R}$, и соответствующий гамильтониан на этом пространстве: $\left[H_{\mathrm{R}}\right]_{\{|\Psi\rangle\} \in\left\{\left|m_{\mathrm{R}}\right\rangle\right\}}=\left[H_{\mathrm{L}}\right]_{\{|\Psi\rangle\} \in\left\{\left|m_{\mathrm{L}}\right\rangle\right\}}$.

2. Второй этап процедуры связан с увеличением размера системы. Так, добавлением одного узла к исходному кластеру L справа образуется кластер S (S - system). Похожим образом присоединением одного узла к кластеру R слева образуется кластер $\mathrm{E}$ ( $\mathrm{E}$ - environment). Размер гильбертова пространства новых блоков равен $M_{\mathrm{S}, \mathrm{E}}=M_{\mathrm{L}, \mathrm{R}} \cdot M_{\mathrm{c}}=64 \times 4=256$, где $M_{\mathrm{c}}-$ количество состояний отдельного узла. Сами базисные состояния „системы“ и „окружения“ формируются как прямые произведения: $\left|m_{\mathrm{S}}\right\rangle=\left|m_{\mathrm{L}}\right\rangle \otimes\left|m_{\mathrm{c}}\right\rangle,\left|m_{\mathrm{E}}\right\rangle=\left|m_{\mathrm{c}}\right\rangle \otimes\left|m_{\mathrm{R}}\right\rangle$. Формальные выражения для гамильтонианов „системы“ и „окружения“" имеют вид

$$
\begin{aligned}
& H_{\mathrm{S}}=H_{\mathrm{L}} \otimes I_{1}+I_{N_{0}} \otimes H_{\mathrm{c}}+H_{\mathrm{S} ; \text { int }} \\
& H_{\mathrm{E}}=H_{\mathrm{c}} \otimes I_{N_{0}}+I_{1} \otimes H_{\mathrm{R}}+H_{\mathrm{E} ; \text { int }} .
\end{aligned}
$$

Здесь посредством $I_{N}$ обозначены единичные операторы в пространстве состояний кластера размером $N$. Операторы $H_{\mathrm{c}}\left(H_{\text {int }}\right)$ могут быть получены из первой (второй) строчки гамильтониана (1) при $N=1(N=2)$. На первой итерации полагается, что

$$
\begin{aligned}
& H_{\mathrm{S}, \text { int }}=H_{\mathrm{S}, \text { int } 0}=I_{N_{0}-1} \otimes H_{\mathrm{int}}, \\
& H_{\mathrm{E}, \text { int }}=H_{\mathrm{E}, \text { int } 0}=H_{\mathrm{int}} \otimes I_{N_{0}-1} .
\end{aligned}
$$

Важно заметить, что добавление узлов именно в центр снимает ряд проблем, связанных с граничными эффектами $[47,48]$.

3. Строится суперкластер $(\mathrm{S}+\mathrm{E})$ размером $2 N_{0}+2$ с числом базисных состояний $M_{\mathrm{S}} \cdot M_{\mathrm{E}}$ :

$$
H=H_{\mathrm{S}} \otimes I_{N_{0}+1}+I_{N_{0}+1} \otimes H_{\mathrm{E}}+I_{N_{0}} \otimes H_{\mathrm{int}} \otimes I_{N_{0}} .
$$

Его диагонализация осуществляется методом Ланцоша [49]. Важным этапом при диагонализации являлось выделение инвариантных подпространств, содержащих четное и нечетное количество фермионов (обозначаются индексами „,+“ и ,-““, соответственно):

$$
H=H_{+} \oplus H_{-} ; \quad H_{ \pm}\left|\Psi_{1,2 ; \pm}\right\rangle=E_{1,2 ; \pm}\left|\Psi_{1,2 ; \pm}\right\rangle .
$$

Здесь явным образом указано, что методом Ланцоша нами находились по два (основное и первое возбужденное) состояния из каждого сектора гильбертова пространства с различной фермионной четностью. На основе найденных четырех многочастичных состояний строится многочастичный оператор плотности суперкластера „система“ + „окружение“ $(\mathrm{S}+\mathrm{E})$ :

$$
\rho=\sum_{j=1,2}\left(p_{j+}\left|\Psi_{j+}\right\rangle\left\langle\Psi_{j+}\left|+p_{j-}\right| \Psi_{j-}\right\rangle\left\langle\Psi_{j-}\right|\right),
$$

где $p_{1+}+p_{2+}+p_{1-}+p_{2-}=1$. В настоящей работе принималось, что

$$
p_{j+}=p_{j-}=1 / 4 \text {. }
$$

Нахождение многочастичной матрицы плотности позволяет извлечь максимально полную информацию о суперкластере. B методе DMRG такая матрица плотности необходима для нахождения редуцированной матрицы плотности с целью корректного учета эффектов границ и квантовой запутанности.

4. Формировалась редуцированная матрица плотности кластера $\mathrm{S}, \rho_{\mathrm{S}}=T r_{\mathrm{E}}|\Psi\rangle\langle\Psi|$, в которой взят парциальный след относительно состояний блока окружения (Е) [50]. В матричной форме имеем

$$
\rho_{\mathrm{s}, \mathrm{s}^{\prime}}=\rho_{\mathrm{s}+, \mathrm{s}^{\prime}+} \oplus \rho_{\mathrm{s}-, \mathrm{s}^{\prime}-} ; \quad \rho_{\mathrm{e}, \mathrm{e}^{\prime}}=\rho_{\mathrm{e}+, \mathrm{e}^{\prime}+} \oplus \rho_{\mathrm{e}-, \mathrm{e}^{\prime}-} .
$$

Здесь состояния системы (окружения) с положительной/отрицательной фермионной четностью обозначены индексами $\mathrm{s} \pm(\mathrm{e} \pm)$. Следовательно, явный вид блоков размерности $M_{\mathrm{S}} / 2 \times M_{\mathrm{S}} / 2=128 \times 128$ редуцированной матрицы плотности для системы $\mathrm{S}$ определяется следующим образом:

$$
\rho_{\mathrm{s} \pm, \mathrm{s}^{\prime} \pm}=\sum_{u=\mathrm{e}_{+}, \mathrm{e}_{-}} \sum_{j=1,2}\left\langle\mathbf{s}_{ \pm}, u \mid \Psi_{j \pm}\right\rangle\left\langle\Psi_{j \pm} \mid \mathbf{s}_{ \pm}^{\prime}\right\rangle .
$$

C целью нахождения эффективного базиса полной системы $\mathrm{S}+\mathrm{E}$, который характеризует ее основные свойства, производится диагонализация редуцированной матрицы плотности: $\rho_{ \pm} \cdot V_{l \pm}=w_{l \pm} \cdot V_{l \pm}$, где $l=1, \ldots, M_{\mathrm{S}} / 2$. Собственные значения $w_{l \pm}$ данной матрицы плотности имеют смысл заселенности квантовых состояний системы $\mathrm{S}$ и обладают свойством $\sum_{l}\left(w_{l+}+w_{l-}\right)=1$. При этом наличие нескольких ненулевых собственных значений $w_{l \pm}$ является индикатором квантовой запутанности системы и ее окружения. Аналогичным образом строится редуцированная матрица плотности окружения $(E)$.

5. Учитывая вышесказанное, квантовые состояния, определяющие эффективные микроскопические степени свободы системы $\mathrm{S}$ определяются собственными векторами $V_{l \pm}$ редуцированной матрицы плотности, имеющими наибольшие параметры заселенности $w_{l \pm}$. Для получения гамильтонианов кластеров L и R, действующих в пространстве этих состояний, строились две матрицы перехода $V_{\mathrm{L} \pm}=\left[V_{1 \pm}, V_{2 \pm}, \ldots, V_{M_{\mathrm{L}} / 2 ; \pm}\right]$ так, что 
соответствующие собственные значения удовлетворяли условиям $w_{1 \pm} \geq w_{2 \pm} \geq \ldots w_{M_{\mathrm{L}} / 2 ; \pm}$. Аналогичным образом строились матрицы перехода $V_{\mathrm{R} \pm}$ для состояний окружения Е. Отметим, что $V_{\mathrm{L}(\mathrm{R})}=\left[V_{\mathrm{L}(\mathrm{R})+}, V_{\mathrm{L}(\mathrm{R})-}\right]$ имеют размерности $M_{\mathrm{S}(\mathrm{E})} \times M_{\mathrm{L}(\mathrm{R})}$. После нахождения матрицы перехода производилась смена представления гамильтонианов $H_{\mathrm{L}}$ и $H_{\mathrm{R}}$, а также соответствующих операторов:

$$
\begin{gathered}
H_{\mathrm{L}(\mathrm{R}) \pm} \rightarrow V_{\mathrm{L}(\mathrm{R}) \pm}^{+} \cdot H_{\mathrm{S}(\mathrm{E}) \pm} \cdot V_{\mathrm{L}(\mathrm{R})} ; \\
A_{\mathrm{L}(\mathrm{R})} \rightarrow V_{\mathrm{S}(\mathrm{E})}^{+} \cdot A_{\mathrm{S}(\mathrm{E})} \cdot V_{\mathrm{S}(\mathrm{E})} ; \\
H_{\mathrm{S}, \text { int } \pm} \rightarrow\left(V_{\mathrm{L}}^{+} \otimes I_{1}\right)_{ \pm}^{+} \cdot\left(I_{1} \otimes H_{\mathrm{S}, \text { int } 0}\right)_{ \pm} \cdot\left(V_{\mathrm{L}}^{+} \otimes I_{1}\right)_{ \pm} ; \\
H_{\mathrm{E}, \text { int } \pm} \rightarrow\left(I_{1} \otimes V_{\mathrm{R}}^{+}\right)_{ \pm}^{+} \cdot\left(H_{\mathrm{E}, \text { int } 0} \otimes I_{1}\right)_{ \pm} \cdot\left(I_{1} \otimes V_{\mathrm{R}}^{+}\right)_{ \pm} .
\end{gathered}
$$

6. Затем, после соответствующей перенормировки состояний, процедура итерировалась с п. 2 до тех пор, пока поведение наблюдаемых характеристик перестанет зависеть от номера итераций.

После достижения сходимости итерационной процедуры поведение физических наблюдаемых отвечает свойствам достаточно большой системы, в которой можно пренебречь влиянием граничных условий. С учетом этого, описанные этапы DMRG процедуры имеют смысл ренормализационной группы для бесконечной системы (infinite DMRG - iDMRG). Характерной ошибкой проведения такой процедуры является так называемая ошибка отсечения: err $=1-\sum_{i=1}^{M_{\mathrm{L}}} w_{i}$, суммирование ведется по собственным значениям матрицы плотности с максимальной величиной. Для проводимых здесь вычислений $\operatorname{err} \propto 10^{-5}$.

Для учета граничных эффектов использовался конечный вариант DMRG процедуры (finite-system DMRG fDMRG). В данном подходе процедура iDMRG продолжается до тех пор, пока размер системы не достигает интересуемого значения в $N$ узлов. После этого сходимость физических характеристик достигается путем проведения последовательности прогонов. Идея заключается в том, чтобы попеременно проводить iDMRG-процедуры только для левой (L) или только для правой $(\mathrm{R})$ половины системы, сохраняя ее размер. При этом необходимые для iDMRG процедуры операторы дополнительной ( $\mathrm{R}$ или $\mathrm{L}$ ) части цепочки сохранять в память и использовать при следующем прогоне.

Итогом выполнения описанного алгоритма являются аппроксимированные значения многочастичных состояний $\left|\Psi_{j \pm}\right\rangle$ и соответствующие уровни энергии $E_{j \pm}$, а также многочастичная матрица плотности (2), позволяющая вычислять средние значения от физических наблюдаемых величин.

\section{3. Анализ полученных результатов}

Рассмотрим вопрос о влиянии сильных локальных взаимодействий: одноузельного $(U)$ и межузельного $(V)$ кулоновского отталкивания на равновесные свойства системы (1). В настоящей работе полагалось, что $U=5$, а параметр $V$ изменялся от нуля до значения $V=3.5$. Из теории сильно коррелированных систем известно, что большие значения одноузельного отталкивания приводят в режиме СЭК, $U \gg 1$, к расщеплению исходной зоны электронов на верхнюю и нижнюю хаббардовские зоны [51]. Между этими зонами на энергетической шкале реализуется мотт-хаббардовская щель, характеризуемая нулевой плотностью электронных состояний. Величина такой щели увеличивается с ростом $U$. При учете сильного межузельного отталкивания $V \gg 1$ происходит аналогичное расщепление каждой из двух хаббардовских зон на две подзоны с перераспределением спектрального веса между ними [52,53].

В настоящей модели (1) эффекты перераспределения спектрального веса между электронными подзонами, индуцированные кулоновскими взаимодействиями, также наблюдаются. Их возникновение можно проследить при построении зависимости средней концентрации электронов на узле $\langle n\rangle=(1 / N) \sum_{f=1}^{N}\left\langle n_{f}\right\rangle$ от химпотенциала системы $\mu$. Такие зависимости приведены на рис. 1 для случаев $V=0(a)$ и $V=3.5(b)$. Видно, что при $U=5$, $V=0$ в зависимости $\langle n\rangle(\mu)$ имеется область $\mu \in[1.5 ; 4]$ с очень малым наклоном, которая является проявлением мотт-хаббардовской щели. Слабый наклон зависимости в этой области связан с наличием в системе потенциала сверхпроводящего спаривания, который обеспечивает существование ненулевой плотности электронных состояний даже в запрещенных зонах спектра электронов без сверхпроводимости.

При учете межузельного отталкивания, $V$, моттхаббардовская щель сохраняется, однако ее положение смещается на интервал значений $\mu \in[7 ; 13]$. Однако в пределах нижней и верхней хаббардовских зон также наблюдается поведение зависимости $\langle n\rangle(\mu)$, аналогичное описанному выше. В частности, рассмотрим нижнюю хаббардовскую зону, которая в дальнейшем будет представлять для нас интерес. В ее пределах, $\mu \in[-2 ; 7]$, наблюдается также возникновение двух подзон в интервалах $\mu \in[-2 ; 1]$ и $\mu \in[3.5 ; 7]$.

Изучение топологических свойств нижней хаббардовской зоны проводилось на основе анализа трех характеристик: спектра возбуждений, майорановской поляризации и спектра квантовой запутанности. Первые две характеристики рассчитывались с использованием fDMRG алгоритма, последняя - на основе алгоритма iDMRG. Спектр возбуждений отсчитывался от основного состояния с энергией $E_{0}=\min \left\{E_{1+}, E_{1-}\right\}$, которая может характеризоваться как положительной, так и отрицательной фермионной четностью. Первые две энергии возбуждений определяются как: $\varepsilon_{j}=E_{j-}-E_{0}$, если $E_{0}=E_{1+}$, и $\varepsilon_{j}=E_{j+}-E_{0}$, если $E_{0}=E_{1-}$. По определению $\varepsilon_{j} \geq 0$, и обращение в ноль данных характеристик может свидетельствовать о реализации в системе квантового перехода. Величина майорановской поляризации 
a

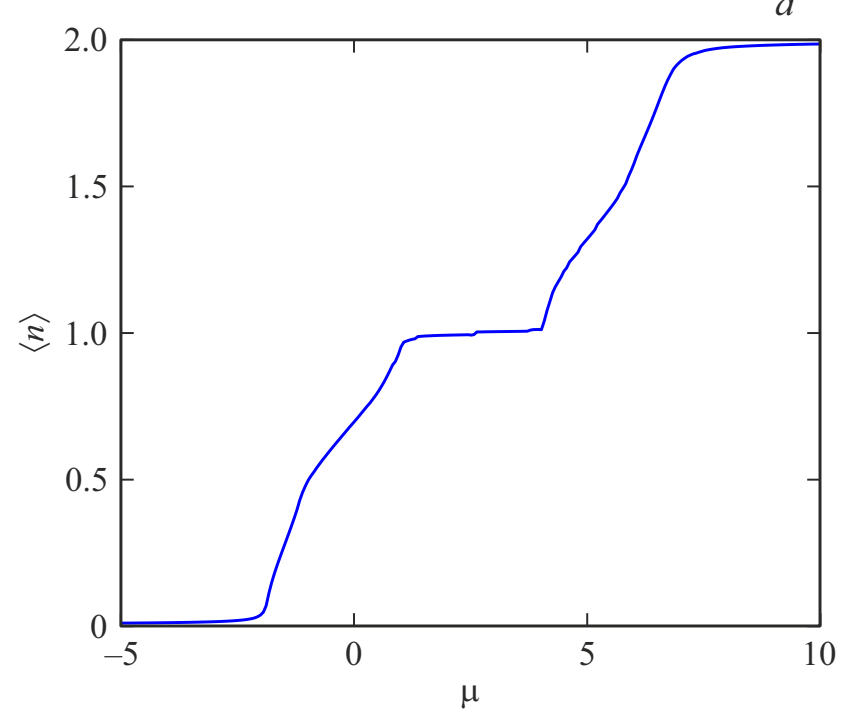

b

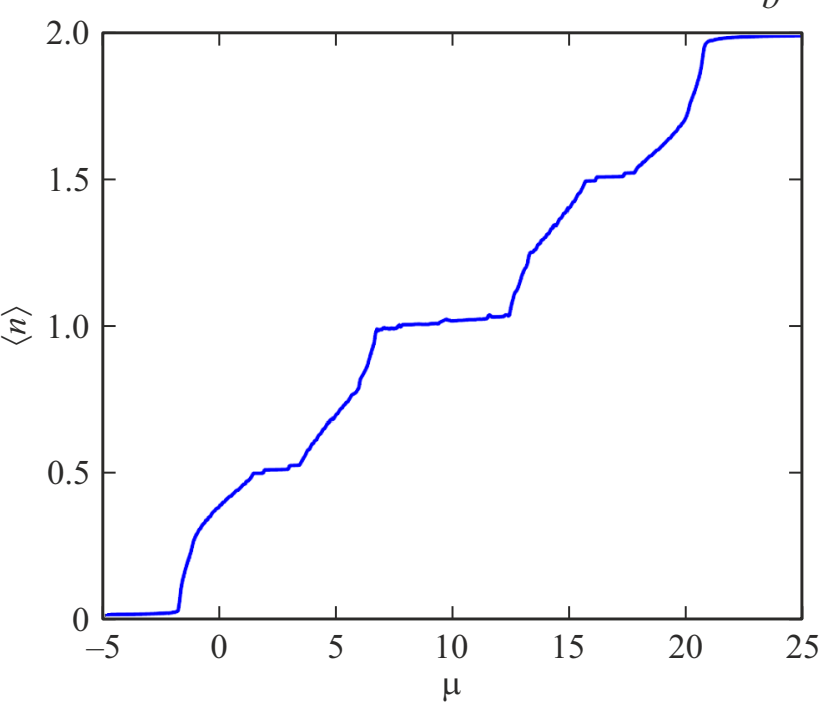

Рис. 1. Зависимость средней концентрации электронов нанопроволоки в режиме сильных электронных корреляций $a$ ) в пренебрежении, $V=0$, и $b$ ) при учете, $V=3.5$, межузельного отталкивания электронов. Остальные параметры системы: $t=1, \alpha=1.5$, $\Delta=-0.5, \Delta_{1}=0.2, h=0.5, U=5, N=100$.

определяется как

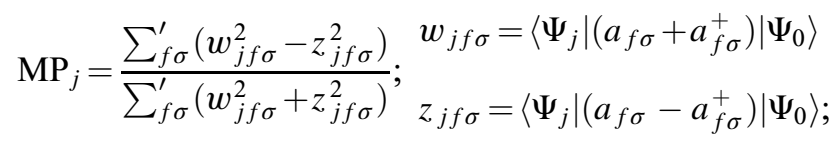

где $\left|\Psi_{0}\right\rangle$ - основное состояние системы: $\left|\Psi_{j}\right\rangle=\left|\Psi_{j \pm}\right\rangle$, если $E_{0}=E_{1 \mp}$. Индекс ' после знака суммы указывает на суммирования по половине узлов СП. Спектр квантовой запутанности $D$ вычислялся по формуле

$$
D=\left[\left(N_{\mathrm{it}}-N_{\mathrm{s}}\right) \cdot \ln (2)\right]^{-1} \cdot \sum_{l=N_{s}}^{N_{\mathrm{it}}} S_{\rho}(l),
$$

где $S_{\rho}(l)=\ln (d(\rho, l)) ; d(\rho, l)$ - кратность вырождения собственных значений редуцированной матрицы плотности (3); $N_{\text {it }}$ - количество итераций алгоритма iDMRG. В расчетах принималось $N_{\text {it }}=400, N_{s}=300$.

Описанные характеристики демонстрируют различное поведение в различных топологических фазах системы. В топологически тривиальной фазе: $\varepsilon_{1,2}>0, \mathrm{MP}_{1,2} \rightarrow 0$, $D \rightarrow 0$. В нетривиальной топологической фазе с одной парой МM: $\varepsilon_{1} \rightarrow 0, \varepsilon_{2}>0, \mathrm{MP}_{1} \rightarrow 1, \mathrm{MP}_{2} \rightarrow 0, D \rightarrow 1$. В нетривиальной фазе с двумя парами ММ: $\varepsilon_{1,2} \rightarrow 0$, $\mathrm{MP}_{1,2} \rightarrow 1, D \rightarrow 2$. Отметим, что вычисление топологического индекса $D$ приводит к однозначным результатам только тогда, когда в системе реализуется электрондырочная симметрия. В случае нарушения такой симметрии поведение $D$ является сильно флуктуирующим (см. рис. $3, b)$.

На рис. 2, $а$ изображена зависимость энергий двух первых возбуждений, $\varepsilon_{1,2}$, от химического потенциала $\mu$ (см. левую ось $y$ ). Поскольку величина одноузельного отталкивания в данном случае существенно превышает все остальные энергетические параметры в системе, то рассматриваемый диапазон значений $\mu$ включает всю нижнюю зону Хаббарда. Другими словами, при $\mu=-2$ имеем $\langle n\rangle \approx 0$ (см. кривую с маркерами-треугольниками и правую ось $y$ ). В свою очередь, $\langle n\rangle \approx 1$ при $\mu=1.5$. Для $\mu>1$ реализуется мотт-хаббардовская щель. Внутри самой зоны выделяются три области с отличающимися топологическими фазами: 1) тривиальная фаза при $\mu \in[-2 ;-1.7] ; 2)$ топологически нетривиальная фаза с двумя майорановскими состояниями при $\mu \in[-1.7 ;-1.2] ; 3)$ топологически нетривиальная фаза с одним майорановским состоянием при $\mu \in[-1.2 ; 1]$. Наличие последних двух фаз следует не только из того, что значения соответствующих энергий обращаются в нуль. Дополнительным важным аргументом выступает поведение характеристик $\mathrm{MP}_{1,2}$ и $D$, представленных на рис. 2, $b$. Оно указывает на краевой характер обоих состояний при $\mu \in[-1.7 ;-1.2]$, где $\mathrm{MP}_{1,2} \rightarrow 1$, и на нелокальность только первого возбуждения при $\mu \in[-1.2 ; 1]$, т.е. $\mathrm{MP}_{1} \rightarrow 1, \mathrm{MP}_{2} \ll 1$ (см. кривые с маркерами-крестиками и кружками соответственно). Из сравнения зависимостей $\varepsilon_{1,2}$ и $\langle n\rangle$ от химического потенциала также следует, что топологические переходы характеризуются особенностями поведения $\partial\langle n\rangle / \partial \mu$. Аналогичные особенности наблюдались в модели цепочки Китаева [54] и модели электронного ансамбля на треугольной решетке в фазе сосуществования сверхпроводимости и магнетизма [55]. Также отметим, что анализ особенностей $\partial\langle n\rangle / \partial \mu$ при $V=0$ позволяет восстановить топологическую фазовую диаграмму системы, приведенную в [41].

На рис. 3, а демонстрируется влияние сильных межузельных кулоновских корреляций на энергии возбужде- 

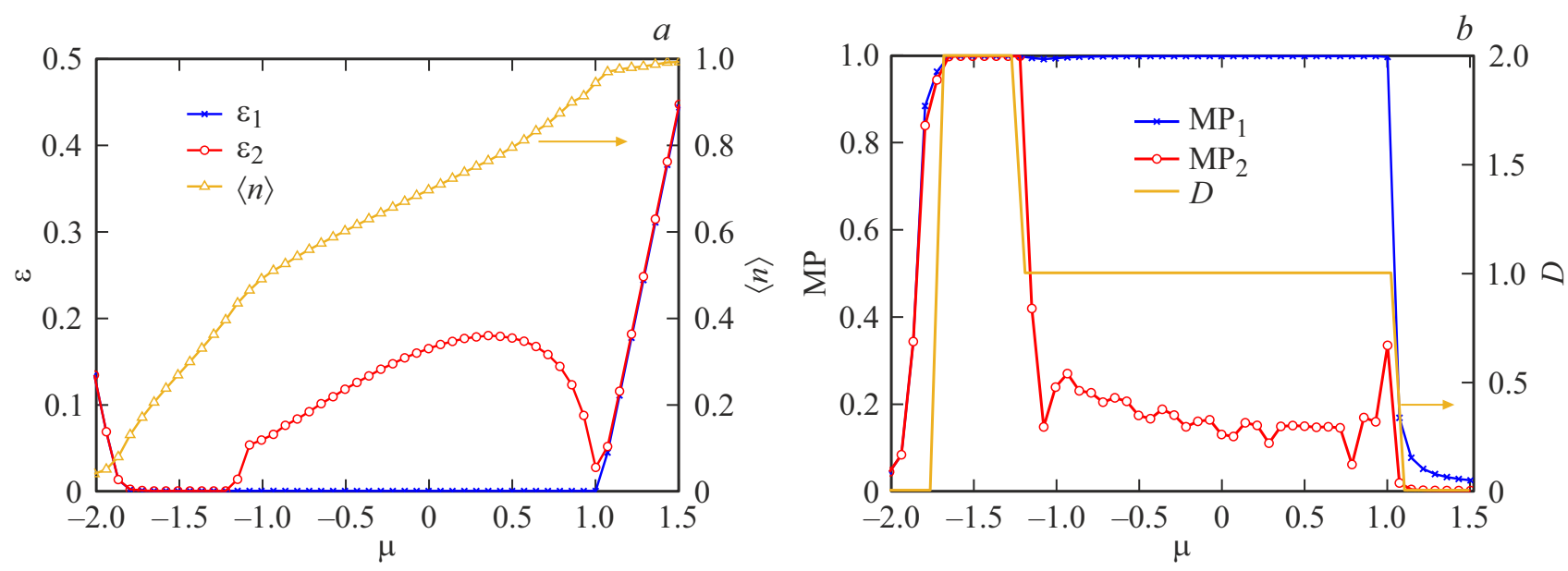

Рис. 2. Зависимость $a$ ) энергий и средней концентрации, а также $b$ ) майорановской поляризации двух первых возбуждений и вырождения спектра запутанности сверхпроводящей проволоки от химического потенциала при $V=0$. Параметры системы соответствуют рис. 1.
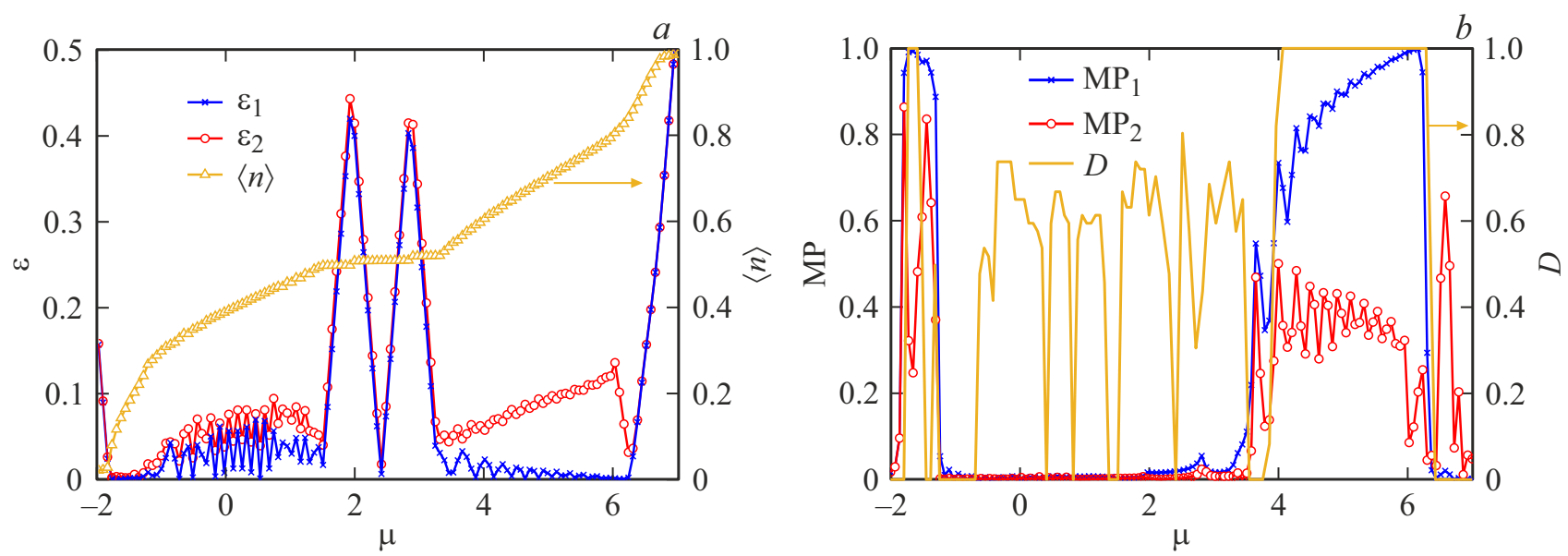

Рис. 3. Влияние сильных межузельных кулоновских корреляций на $a$ ) энергии возбуждения и среднюю концентрацию, а также $b)$ майорановскую поляризацию и вырождение спектра запутанности сверхпроводящей проволоки для параметров рис. 2 и $V=3.5$.

ния и среднюю одноузельную концентрацию в нижней хаббардовской зоне. Видно, что при большом межузельном отталкивании внутри нее образуются две подзоны при $\mu \in[-2 ; 1.5]$ и $\mu \in[3 ; 7]$. В щели между ними концентрация выходит на плато, где $\langle n\rangle \approx 0.5$ (см. кривую с треугольными маркерами и ось ординат справа). С ростом $V$ топологическая фаза с одним майорановским состоянием начинает быстро подавляться для большинства значений $\mu$. Этот эффект значительно сильнее, чем обсуждавшийся ранее в проволоках класса BDI, вызванный исключительно сильным хаббардовским отталкиванием [41]. Существенно, что при $\mu \in[-1.9 ;-1.5]$ и $\mu \in[4 ; 6]$ реализация одиночных майорановских состояний все-таки возможна. Данное наблюдение подтверждается также расчетами майорановской поляризации $\mathrm{MP}_{1,2}$ и топологического индекса $D$, которые представлены на рис. $3, b$. Такую устойчивость топологических фаз по отношению к сильным зарядовым корреляци- ям можно объяснить тем, что отмеченные области химпотенциала характеризуются малой концентрацией электронов или дырок, и, соответственно, относительно слабым влиянием межузельного отталкивания на свойства системы.

Заметим также, что при наличии сильного межузельного отталкивания $V=3.5$ на рис. $3, b$ в интервале значений химпотенциала $\mu \in[-1.5 ; 3.5]$ характеристика $D$ демонстрирует сильно флуктуирующее поведение. Последнее связано с эффективным подавлением ближних сверхпроводящих спариваний и нарушением электрондырочной симметрии в системе. Аналогичный эффект наблюдается в случае $\Delta_{1}=0, V=0$ и очень высокими значениями хаббардовского отталкивания $U>10$. Видно, что учет межузельного отталкивания приводит к много более быстрому подавлению сверхпроводимости в системе по сравнению со случаем учета только хаббардовского отталкивания. 
В заключение отметим, что при попадании химпотенциала в индуцированные кулоновскими взаимодействиями квазизапрещенные зоны в системе может происходить квантовый фазовый переход без изменения топологического индекса (см. зависимости $\varepsilon_{1,2}(\mu)$ на рис. $3, a$ в окрестности $\mu \approx 2.2$ ). Важно, что такие квантовые переходы обусловлены именно наличием в системе межузельного отталкивания и не могут быть найдены при $V=0$. Однако выяснение природы и свойств таких квантовых переходов выходит за пределы настоящего исследования.

\section{4. Заключение}

В работе на основе метода ренорм-группы для матрицы плотности исследовано влияние сильного межузельного отталкивания электронов на топологические фазы сверхпроводящей нанопроволоки со спин-орбитальным взаимодействием и наведенной сверхпроводимостью расширенного $s$-типа симметрии, помещенной во внешнее магнитное поле. Проведенный анализ является продолжением исследования [41], в котором сверхпроводящая нанопроволока характеризовалась сильным хаббардовских отталкиванием, тогда как межузельные кулоновские корреляции считались значительно экранированными. Показано, что с ростом интенсивности отталкивания электронов, находящихся на соседних узлах, происходит расщепление нижней хаббардовской зоны на две подзоны, с возникновением на энергетической шкале области с очень малой плотностью состояний, аналогичной мотт-хаббардовской щели. Возникновение двух подзон наблюдается и при концентрациях выше единицы. При этом в каждой из энергетических областей с малой плотностью состояний (как в моттхаббардовской щели, так и в индуцированных межузельным отталкиванием щелях) при изменении химпотенциала системы наблюдаются квантовые переходы без изменения топологического индекса. Такие переходы реализуются только при наличии достаточно сильного межузельного отталкивания. Топологические свойства описанных электронных подзон в нижней зоне Хаббарда исследовались на основе анализа спектра возбуждений, майорановской поляризации, а также спектра квантовой запутанности. Показано, что при сильном межузельном отталкивании нетривиальные топологические фазы могут индуцироваться только в параметрических областях с низкой концентрацией электронов или дырок. При остальных заполнениях системы носителями реализуется топологически тривиальная фаза с подавленной за счет кулоновских взаимодействий сверхпроводимостью. При этом замечено, что межузельное отталкивание, как фактор, приводящий к разрушению куперовского спаривания, является существенно более сильным, чем хаббардовское взаимодействие.

\section{Финансирование работы}

Работа выполнена при поддержке Российского фонда фундаментальных исследований (проекты № 19-0200348, 20-32-70059), Правительства Красноярского края и Краевого фонда науки (проекты № 20-42-243001, 20-42-243005). ШМС выражает благодарность Фонду развития теоретической физики и математики „БАЗИС“. АСВ благодарит за поддержку грант Президента РФ MK-1641.2020.2.

\section{Конфликт интересов}

Авторы заявляют, что у них нет конфликта интересов.

\section{Список литературы}

[1] J. Chen, B.D. Woods, P. Yu, M. Hocevar, D. Car, S.R. Plissard, E.P.A.M. Bakkers, T.D. Stanescu, S.M. Frolov. Phys. Rev. Lett. 123, 10, 107703 (2019).

[2] H. Pan, W.S. Cole, J.D. Sau, S. Das Sarma. Phys. Rev. B 101, 2, 024506 (2020).

[3] S.R. Elliott, M. Franz. Rev. Mod. Phys. 87,‘1, 137 (2015).

[4] В.В. Вальков, В.А. Мицкан, А.О. Злотников, М.С. Шустин, С.В. Аксенов. Письма в ЖЭТФ 110, 2, 126 (2019).

[5] В.В. Вальков, М.С. Шустин, С.В. Аксенов, А.О. Злотников, А.Д. Федосеев, В.А. Мицкан, М.Ю. Каган. УФН (2021). В печати. DOI: 0.3367/UFNr.2021.03.038950.

[6] M. Deng, S. Vaitiekénas, E. Prada, P. San-Jose, J. Nygård, P. Krogstrup, R. Aguado, C.M. Marcus. Phys. Rev. B 98, 8, 085125 (2018)

[7] V.V. Val'kov, M.Yu. Kagan, S.V. Aksenov. J. Phys.: Condens. Matter 31, 22, 225301 (2019).

[8] С.В. Аксенов, М.Ю. Каган. Письма в ЖЭТФ 111, 5, 321 (2020).

[9] D. Sticlet, C. Bena, P. Simon. Phys. Rev. Lett. 108, 9, 096802 (2012).

[10] V.V. Val'kov, S.V. Aksenov. J. Magn. Magn. Mater. 440, 112 (2017).

[11] В.В. Вальков, С.В. Аксенов. ФНТ 43, 4, 546 (2017).

[12] V.V. Val'kov, S.V. Aksenov. J. Magn. Magn. Mater. 465, 88 (2018).

[13] В.В. Вальков, В.А. Мицкан, М.С. Шустин. Письма в ЖЭТФ 106, 12, 762 (2017).

[14] В.В. Вальков, В.А. Мицкан, М.С. Шустин. ЖЭТФ 156, 3, 507 (2019).

[15] A.P. Schnyder, S. Ryu, A. Furusaki, A.W.W. Ludwig. Phys. Rev. B 78, 19, 195125 (2008).

[16] A. Kitaev. AIP Conf. Proc. 1134, 22 (2009).

[17] О. Зайцев. Исследование перехода металл-диэлектрик. Препринт Института атомной энергии им. И.В. Курчатова. № ИАЭ-3927/1 (1984).

[18] Y. Sato, S. Matsuo, C.-H. Hsu, P. Stano, K. Ueda, Y. Takeshige, H. Kamata, J.S. Lee, B. Shojaei, K. Wickramasinghe, J. Shabani, C. Palmstrøm, Y. Tokura, D. Loss, S. Tarucha. Phys. Rev. B 99, 15, 155304 (2019).

[19] S.I. Tomonaga. Prog. Theor. Phys. 5, 4, 544 (1950).

[20] J.M. Luttinger. J. Math. Phys. 4, 9, 1154 (1963).

[21] M. Bockrath, D.H. Cobden, J. Lu, A.G. Rinzler, R.E. Smalley, L. Balents, P.L. McEuen. Nature 397, 598 (1999).

[22] C.L.M. Wong, K.T. Law. Phys. Rev. B 86, 18, 184516 (2012). 
[23] M. Thakurathi, P. Simon, I. Mandal, J. Klinovaja, D. Loss. Phys. Rev. B 97, 4, 045415 (2018).

[24] A. Haim, A. Keselman, E. Berg, Y. Oreg. Phys. Rev. B 89, 22 , 220504(R) (2014).

[25] E. Fradkin, L.P. Kadanoff. Nucl. Phys. B 170, 1, 1 (1980).

[26] P. Fendley. J. Stat. Mech. 2012, 11020 (2012).

[27] L. Fidkowski, A. Kitaev. Phys. Rev. B 81, 13, 134509 (2010).

[28] A.Y. Kitaev. Phys. Usp. 44, 10S, 131 (2001).

[29] H. Katsura, D. Schuricht, M. Takahashi. Phys. Rev. B 92, 11, 115137 (2015).

[30] G. Kells. Phys. Rev. B 92, 8, 081401(R) (2015).

[31] J. Boeyens, I. Snyman. Phys. Rev. B 102, 2, 024513 (2020).

[32] P. Fendley. J. Phys. A 49, 30LT1 (2016).

[33] D.A. Ivanov. Phys. Rev. Lett. 86, 2, 268 (2001).

[34] Z.-T. Zhang, F. Mei, X.-G. Meng, B.-L. Liang, Z.-S. Yang. Phys. Rev. A 100, 1, 012324 (2019).

[35] H.-L. Lai, P.-Y. Yang, Y.-W. Huang, W.-M. Zhang. Phys. Rev. B 97, 5, 054508 (2018).

[36] Y. Peng, F. Pientka, L.I. Glazman, F. von Oppen. Phys. Rev. Lett. 114, 10, 106801 (2015).

[37] H.T. Ng. Sci. Rep. 5, 12530 (2015).

[38] A. Wieckowski, A. Ptok. Phys. Rev. B 100, 14, 144510 (2019).

[39] A.M. Turner, F. Pollmann, E. Berg. Phys. Rev. B 83, 7, 075102 (2011).

[40] E.M. Stoudenmire, J. Alicea, O.A. Starykh, M.P.A. Fisher. Phys. Rev. B 84, 1, 014503 (2011).

[41] S.V. Aksenov, A.O. Zlotnikov, M.S. Shustin. Phys. Rev. B 101, 12, 125431 (2020).

[42] А.О. Злотников, С.В. Аксенов, М.С. Шустин. ФТТ 62, 9, 1447(2020).

[43] S.R. White, R.M. Noack. Phys. Rev. Lett. 68, 24, 3487 (1992).

[44] S.R. White. Phys. Rep. 301, 1, 187 (1998).

[45] S.R. White, R.M. Noack. In: Density matrix renormalization: a new numerical method in physic/Eds I. Peschel, X. Wang, M. Kaulke, K. Hallberg. Springer, Berlin (1999).

[46] K. Wilson. Rev. Mod. Phys. 47, 4, 773 (1975).

[47] S.R. White. Phys. Rev. Lett. 69, 19, 2863 (1992).

[48] U. Schollwock. Rev. Mod. Phys. 77, 1, 259 (2005).

[49] G. Golub, C.F. van Loan. Matrix computations. The John Hopkins University Press, Baltimore (2013).

[50] К. Коэн-Таннуджи, Б. Диу, Ф. Лалоэ (С. Cohen-Tannoudji, B. Diu, F. Laloe). Квантовая механика..Изд-во Уральского ун-та, Екатеринбург (2000). Т. 1.

[51] J.C. Hubbard. Proc. Roy. Soc. London A 276, 1365, 238 (1963).

[52] В.В. Вальков, М.М. Коровушкин. ЖЭТФ 139, 1, 126 (2011).

[53] V. Val'kov, M. Korovushkin. J. Phys. Soc. Jpn 80, 1, 014703 (2011).

[54] Y.-H. Chan, C.-K. Chiu, K. Sun. Phys. Rev. B 92, 10, 104514 (2015).

[55] В.В. Вальков, А.О. Злотников. Письма в ЖЭТФ 104, 7, 512 (2016).

Редактор Е.В. Толстякова 